[Article]

\title{
水热法合成高功率锂离子二次电池用球形 $\mathrm{Li}_{4} \mathrm{Ti}_{5} \mathrm{O}_{12}$ 负极材料
}

\author{
问慧张欢张鼎朱智 其 鲁*
}

(北京大学化学与分子工程学院, 应用化学系, 新能源材料与技术实验室, 北京分子科学国家实验室, 北京 100871)

\begin{abstract}
摘要: 以球形 $\mathrm{TiO}_{2}$ 和 $\mathrm{LiOH}$ 溶液为反应物, 通过水热法合成了尖晶石型 $\mathrm{Li}_{4} \mathrm{Ti}_{5} \mathrm{O}_{12}$, 并使用 $X$ 射线衍射 $(\mathrm{XRD})$ 、傅 里叶变换红外 (FTIR)光谱、扫描电镜(SEM)和激光粒度分布(PSD)对其结构、形貌和电化学性能进行了表征. 结 果表明: 通过该法得到的产品颗粒大小均匀, 粒度分布狭窄, 结构与标准 $\mathrm{Li}_{4} \mathrm{Ti}_{5} \mathrm{O}_{12}$ 谱图一致. 实验选定温度下所 得的 $\mathrm{Li}_{4} \mathrm{Ti}_{5} \mathrm{O}_{12}$ 均表现出良好的电化学性能. 其中, $800{ }^{\circ} \mathrm{C}$ 热处理所得样品的电化学性能最好, 室温下, 以 35 $\mathrm{mA} \cdot \mathrm{g}^{-1}$ 的电流密度进行充放电, 其可逆容量达到 $162 \mathrm{mAh} \cdot \mathrm{g}^{-1}$, 同时这种材料也表现出良好的倍率性能, 即使 在 $720 \mathrm{~mA} \cdot \mathrm{g}^{-1}$ 的电流密度条件下进行充放电, 其可逆容量仍可达到 $124 \mathrm{mAh} \cdot \mathrm{g}^{-1}$.
\end{abstract}

关键词：水热法合成；球形； $\mathrm{Li}_{4} \mathrm{Ti}_{5} \mathrm{O}_{12} ;$ 电化学性能; 锂离子二次电池

中图分类号: 0646

\section{Hydrothermal Synthesis of Spherical $\mathrm{Li}_{4} \mathrm{Ti}_{5} \mathrm{O}_{12}$ as Anode Material for High Power Lithium-Ion Secondary Battery}

\author{
YAN Hui $\quad$ ZHANG Huan $\quad$ ZHANG Ding ZHU Zhi QI Lu* \\ (Beijing National Laboratory for Molecular Sciences, New Energy Materials and Technology Laboratory, Department of \\ Applied Chemistry, College of Chemistry and Molecular Engineering, Peking University, Beijing 100871, P. R. China)
}

\begin{abstract}
Pure spinel-type lithium titanate, $\mathrm{Li}_{4} \mathrm{Ti}_{5} \mathrm{O}_{12}$, was successfully fabricated by a facile hydrothermal route using anatase $\mathrm{TiO}_{2}$ and $\mathrm{LiOH}$ solution as raw materials. The obtained samples were characterized by X-ray diffraction (XRD), Fourier transform infrared (FTIR) spectroscopy, scanning electron microscopy (SEM), and laser particle size distribution (PSD) analysis. The results showed that a spherical well-crystallized $\mathrm{Li}_{4} \mathrm{Ti}_{5} \mathrm{O}_{12}$ oxide was obtained at a calcination temperature of $800{ }^{\circ} \mathrm{C}$. The optimal $\mathrm{Li}_{4} \mathrm{Ti}_{5} \mathrm{O}_{12}$ also has excellent electrochemical performance, which reached $162 \mathrm{mAh} \cdot \mathrm{g}^{-1}$ at a current density of $35 \mathrm{~mA}$. $\mathrm{g}^{-1}$ and a good rate capability with a capacity reached $124 \mathrm{mAh} \cdot \mathrm{g}^{-1}$ even at a current density of $720 \mathrm{~mA} \cdot \mathrm{g}^{-1}$.
\end{abstract}

Key Words: Hydrothermal synthesis; Spherical; $\mathrm{Li}_{4} \mathrm{Ti}_{5} \mathrm{O}_{12} ; \quad$ Electrochemical performance;

Lithium-ion secondary battery

\section{1 引言}

目前以碳质材料为负极的小型锂离子电池已 广泛应用于手机、数码相机和笔记本电脑等小型数 码产品, 但由于这些小型锂电池在快速充放电过程 中, 锂离子的不可逆成分迅速增加, 阻碍了电池的 大电流充放电能力, 缩短了使用寿命, 并容易引发
燃烧、爆炸等安全性问题, 严重限制了其在大型用 电设备如电动汽车和电力储能设备等中的应用. 近 年来, 众多研究者积极寻找性能优良能够替代碳材 料的负极材料, 在各种可替代的材料中, ${ }^{1-5}$ 具有较高 嵌锂电位的 $\mathrm{Li}_{4} \mathrm{Ti}_{5} \mathrm{O}_{12}\left(1.5 \mathrm{~V} v s \mathrm{Li} / \mathrm{Li}^{+}\right)$材料拥有独特 的物理化学性质, 尤其是其极佳的循环稳定性, 在

Received: March 28, 2011; Revised: May 17, 2011; Published on Web: July 4, 2011.

"Corresponding author. Email: qilu@pku.edu.cn; Tel: +86-10-62751000.

The project was supported by the National High Technology Research and Development Program of China (863) (2008AA11A102).

国家高技术研究发展计划项目(863) (2008AA11A102)资助

(C) Editorial office of Acta Physico-Chimica Sinica 
多次充放电过程中晶胞常数几乎不发生任何变化, 因此成为人们关注的焦点.

研究表明, 尽管 $\mathrm{Li}_{4} \mathrm{Ti}_{5} \mathrm{O}_{12}$ 材料具有循环充放电 使用寿命长、安全性好等特点, 但它低的导电率、锂 离子扩散速率以及低密度制约了其大规模的应用. 通过对 $\mathrm{Li}_{4} \mathrm{Ti}_{5} \mathrm{O}_{12}$ 进行掺杂和导电包覆,, 7 虽然加快了 锂离子的扩散速率, 获得倍率性能更好的材料. 但 材料的粒径和形貌同样影响电池的电化学性能. 微 米甚至纳米级颗粒可以缩短锂离子在材料中的扩 散路径, 增加材料的容量, 提高材料的倍率性能. ${ }^{8-10}$ 目前, $\mathrm{Li}_{4} \mathrm{Ti}_{5} \mathrm{O}_{12}$ 的制备方法主要是固相法 ${ }^{11-13}$ 和液相 法., ${ }^{9} 10,14-19$ 其中, 固相法适合大规模生产, 但所得材料 颗粒尺寸大且分布不均匀; 溶胶-凝胶法、微乳液法 和喷雾沉积法等液相法可以制备尺寸可控的小粒 径材料, 但其步骤繁琐、时间较长且所用原料对环 境有污染.

本文以锐钛矿型 $\mathrm{TiO}_{2}$ 和 $\mathrm{LiOH}$ 溶液为反应物, 通过水热反应得到中间体产物, 之后进行高温热 处理得到球形 $\mathrm{Li}_{4} \mathrm{Ti}_{5} \mathrm{O}_{12}$. 对所得的材料进行了 $\mathrm{X}$ 射 线衍射(XRD)、傅里叶变换红外(FTIR)光谱、扫描电 镜(SEM)、激光粒度分布(PSD) 等分析测试, 并将其 装配成 R2032 型扣式电池进行了电化学性能的测 试.

\section{2 实验部分}

\section{$2.1 \mathrm{Li}_{4} \mathrm{Ti}_{5} \mathrm{O}_{12}$ 的合成}

\subsection{1 固相法合成 $\mathrm{Li}_{4} \mathrm{Ti}_{5} \mathrm{O}_{12}$}

按化学计量比称取 $\mathrm{LiOH} \cdot \mathrm{H}_{2} \mathrm{O}$ (分析纯, 汕头市 西陇化工厂有限公司)与 $\mathrm{TiO}_{2}$ (优级, 上海跃江钛白 化工制品有限公司)在玛瑙研钭中, 加入适量的无水 乙醇研磨 $1 \mathrm{~h}$, 随后再置于 $100{ }^{\circ} \mathrm{C}$ 烘箱中干燥后再研 磨 10-15 min. 将所得混合物于 $500{ }^{\circ} \mathrm{C}$ 下进行热处 理 $6 \mathrm{~h}$ 后取出, 加入适当无水乙醇进行研磨 $30 \mathrm{~min}$. 随后再置于 $100^{\circ} \mathrm{C}$ 烘箱中干燥后研磨 10-15 min. 将所得样品于 $850^{\circ} \mathrm{C}$ 高温下处理 2-15 h.

\section{1 .2 水热法合成 $\mathrm{Li}_{4} \mathrm{Ti}_{5} \mathrm{O}_{12}$}

硫酸钛(化学纯, 国药集团化学试剂有限公司) 溶于蒸馏水, 得到 $0.2 \mathrm{~mol} \cdot \mathrm{L}^{-1}$ 的溶液, 将硫酸钛溶液 与正丙醇(分析纯, 北京益利精细化学品有限公司) 以 $1: 1$ (体积比)的比例混匀, 之后将其在 $80^{\circ} \mathrm{C}$ 的水 浴中水解得到球形 $\mathrm{TiO}_{2}{ }^{20}$

称取一定量的 $\mathrm{LiOH} \cdot \mathrm{H}_{2} \mathrm{O}$ (分析纯, 汕头市西陇 化工厂有限公司) 溶于蒸馏水得到氢氧化锂溶液. 按
化学计量比称取自制的球形 $\mathrm{TiO}_{2}$, 将其与氢氧化锂 溶液在水热反应釜中混合均匀, 将反应釜置于 $100^{\circ} \mathrm{C}$ 的烘箱中反应 $20 \mathrm{~h}$.

将水热反应所得的中间体研磨, 置于马弗炉, 空气中 $800^{\circ} \mathrm{C}$ 热处理 $2 \mathrm{~h}$, 得到 $\mathrm{Li}_{4} \mathrm{Ti}_{5} \mathrm{O}_{12}$ 样品.

\section{$2.2 \mathrm{Li}_{4} \mathrm{Ti}_{5} \mathrm{O}_{12}$ 的物理化学性能测试}

通过 X 射线粉末衍射仪 (XRD, Rigaku, D/ max-2500, 日本)对产物的晶体结构进行测定, 测试 条件为 $\mathrm{Cu} K_{a}$ 靶, 石墨单色器, 扫描速率为 $5\left(^{\circ}\right)$. $\mathrm{min}^{-1}$, 扫描范围为 $10^{\circ}-80^{\circ}$. 通过红外光谱仪(FTIR8400 , SHIMADZU, 日本)对反应物、水热反应中间 体和产物进行了红外光谱分析. 产物的形貌采用扫 描电镜(SEM, JSM-5600LV, 日本)进行表征. 产物粒 径分析通过激光粒度分析仪(PSD, LS 230, 美国 BECKMAN COULTER)进行.

\section{$2.3 \mathrm{Li}_{4} \mathrm{Ti}_{5} \mathrm{O}_{12}$ 电池的制备}

将 $\mathrm{Li}_{4} \mathrm{Ti}_{5} \mathrm{O}_{12}$ 、导电剂、聚偏氟乙烯(PVDF) 按 90 : 4:6 的质量比混合均匀, 搅拌成一定粘度的电极浆 料, 涂覆在铝䇴上, 经过烘干、碾压、裁剪等步骤制 成研究电极. 以金属锂作为对电极, $1 \mathrm{~mol} \cdot \mathrm{L}^{-1}$ 的 $\mathrm{LiPF}_{6}$ /碳酸乙烯酯 $(\mathrm{EC})+$ 碳酸二甲酯 $(\mathrm{DMC})+$ 碳酸甲 乙酯 $(\mathrm{EMC})$ (质量比 $1: 1: 1)$ 溶液为电解液, 在充满氩 气的手套箱中组装成 $R 2032$ 型两电极电池.

\section{4 电化学性能测试}

将组装好的电池于 $25^{\circ} \mathrm{C}$ 下放置 $24 \mathrm{~h}$, 采用电 池测试仪(Land-2001A, 武汉金诺电子有限公司)进 行恒流充放电, 评价电池的充放电性能、功率性能 和循环性能等, 充放电电压范围为 2.5-1.0 V (vs Li/ $\mathrm{Li}^{+}$).

\section{3 结果与讨论}

\section{1 $\mathrm{Li}_{4} \mathrm{Ti}_{5} \mathrm{O}_{12}$ 固相合成法与水热合成法的比较}

图 1 为 $\mathrm{TiO}_{2}$ 和 $\mathrm{LiOH} \cdot \mathrm{H}_{2} \mathrm{O}$ 反应混合物及其在不 同温度下热处理不同时间的 XRD 图, 从图中的(a) 曲线可以看出, 在 $25.3^{\circ}$ 处出现了 $\mathrm{TiO}_{2}$ 的强衍射峰, $30^{\circ}-35^{\circ}$ 之间出现了一系列非常弱的 $\mathrm{LiOH} \cdot \mathrm{H}_{2} \mathrm{O}$ 衍 射峰; 反应物经 $500{ }^{\circ} \mathrm{C}$ 处理 $6 \mathrm{~h}$ 后出现了 $\mathrm{Li}_{4} \mathrm{Ti}_{5} \mathrm{O}_{12}$ 相, 但仍有未反应的 $\mathrm{TiO}_{2}$ 等原料存在, 在 $500{ }^{\circ} \mathrm{C}$ 处 理 $6 \mathrm{~h}$ 的基础上再经过 $850{ }^{\circ} \mathrm{C}$ 处理才形成完整的尖 晶石相, 且热处理时间大于 $10 \mathrm{~h}$ 所得产物的 XRD 图 才无明显的杂质峰.

图 2 为水热法合成的中间体经不同热处理温度 所得的 $\mathrm{Li}_{4} \mathrm{Ti}_{5} \mathrm{O}_{12}$ 的 XRD 图谱. 从图中可以看出, 三 


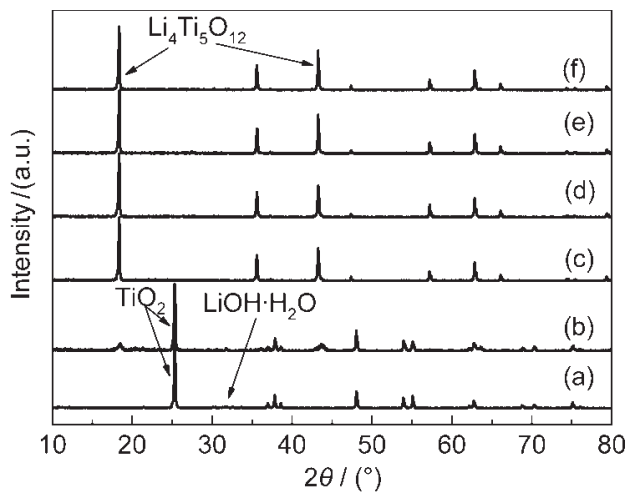

图 1 固相法合成 $\mathrm{Li}_{4} \mathrm{Ti}_{5} \mathrm{O}_{12}$ 的 XRD 谱图

Fig.1 XRD patterns of the $\mathrm{Li}_{4} \mathrm{Ti}_{5} \mathrm{O}_{12}$ obtained after solid-state reaction

(a) $\mathrm{TiO}_{2}$ and $\mathrm{LiOH} \cdot \mathrm{H}_{2} \mathrm{O}$ raw materials, (b) $500{ }^{\circ} \mathrm{C}, 6 \mathrm{~h}$, (c) b+850 ${ }^{\circ} \mathrm{C}$, $2 \mathrm{~h},(\mathrm{~d}) \mathrm{b}+850{ }^{\circ} \mathrm{C}, 5 \mathrm{~h},(\mathrm{e}) \mathrm{b}+850^{\circ} \mathrm{C}, 10 \mathrm{~h}$, (f) b+850 ${ }^{\circ} \mathrm{C}, 15 \mathrm{~h}$

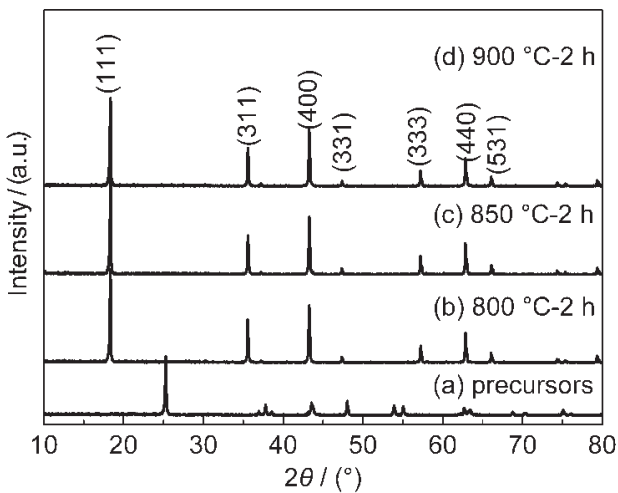

图 2 中间体分别经不同温度热处理所得 XRD 谱图

Fig.2 XRD patterns of the precursors after calcination at different temperatures

(a) precursors; $T /{ }^{\circ} \mathrm{C}$ : (b) 800, (c) 850, (d) 900

个不同热处理温度下所得材料的 XRD 谱图几乎一 样, 其 $2 \theta$ 值分别在 $18.4^{\circ} 、 35.6^{\circ} 、 43.3^{\circ} 、 47.4^{\circ} 、 57.2^{\circ}$ 、 $62.8^{\circ}$ 和 $66.1^{\circ}$ 等处出现了衍射峰, 与标准 $\mathrm{Li}_{4} \mathrm{Ti}_{5} \mathrm{O}_{12}$ 的 $\mathrm{XRD}$ 衍射峰一致. 这表明产物具有单一的立方尖晶 石相结构, 未出现杂质相. 结果表明, $800{ }^{\circ} \mathrm{C}$ 处理 $2 \mathrm{~h}$ 便可得到纯相的结晶度较好的 $\mathrm{Li}_{4} \mathrm{Ti}_{5} \mathrm{O}_{12}$ 材料, 在这 样低的热处理温度和短的热处理时间下就可得到 高纯度、无杂质的 $\mathrm{Li}_{4} \mathrm{Ti}_{5} \mathrm{O}_{12}$, 说明水热反应法对于合

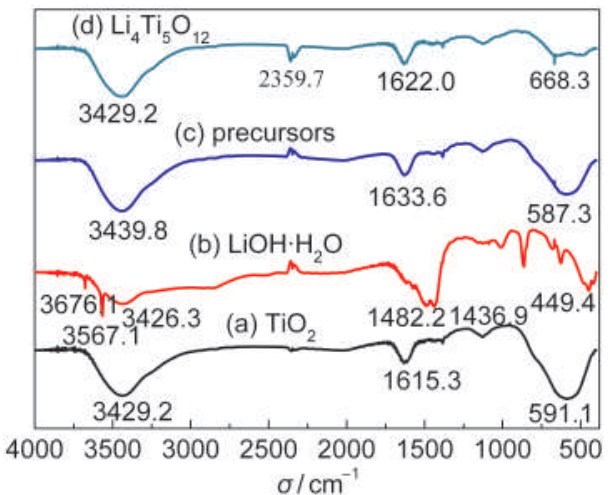

图 3 样品的 FTIR 谱图

Fig.3 FTIR spectra of samples

(a) $\mathrm{TiO}_{2}$, (b) $\mathrm{LiOH} \cdot \mathrm{H}_{2} \mathrm{O}$, (c) precursors, (d) $\mathrm{Li}_{4} \mathrm{Ti}_{5} \mathrm{O}_{12}$

成纯相的钛酸锂材料是有效的.

\subsection{FTIR 光谱分析}

图 3 所示为水热反应中的反应物、中间体和 $800{ }^{\circ} \mathrm{C}$ 热处理中间体 $2 \mathrm{~h}$ 所得的 $\mathrm{Li}_{4} \mathrm{Ti}_{5} \mathrm{O}_{12}$ FTIR 谱 图. 由图可见, $\mathrm{TiO}_{2}$ 和 $\mathrm{LiOH}$ 溶液进行水热反应后所 得的中间体与 $\mathrm{TiO}_{2}$ 谱图无明显区别, 但无 $\mathrm{LiOH}$ 的 吸收带. 经 $800{ }^{\circ} \mathrm{C}$ 处理后所得样品的红外光谱在 2359.7 和 $668.3 \mathrm{~cm}^{-1}$ 处出现两个吸收峰, 分别对应尖 晶石中 $\mathrm{Ti}-\mathrm{O}$ 键和 $\mathrm{MO}_{6}\left(\mathrm{TiO}_{6}\right)$ 八面体的伸缩振动. ${ }^{17,21}$ $1622.0 \mathrm{~cm}^{-1}$ 处的吸收峰是由材料吸附了空气中的 $\mathrm{CO}_{2}$ 引起的, 由于 $\mathrm{Li}_{4} \mathrm{Ti}_{5} \mathrm{O}_{12}$ 材料吸附了水, 所以在 $3429.2 \mathrm{~cm}^{-1}$ 附近出现了吸收峰. ${ }^{17,22}$

\section{3 $\mathrm{Li}_{4} \mathrm{Ti}_{5} \mathrm{O}_{12}$ 的表面形貌分析}

图 4 是自制球形 $\mathrm{TiO}_{2}$ 和经过不同热处理温度所 得的 $\mathrm{Li}_{4} \mathrm{Ti}_{5} \mathrm{O}_{12}$ 的 $\mathrm{SEM}$ 照片. 从图中可以很明显地看 到, 随着热处理温度的逐渐升高, 所得材料的颗粒 尺寸逐渐增大. 不同的热处理温度下, 所得材料粒 径分布均匀一致, 集中在 $1 \mu \mathrm{m}$ 左右, $900{ }^{\circ} \mathrm{C}$ 处理所 得样品有轻微的烧结现象, 这表明 $\mathrm{Li}_{4} \mathrm{Ti}_{5} \mathrm{O}_{12}$ 的平均 颗粒大小和粒径分布是由热处理温度决定的, 在较 高温度下材料容易烧结长大. SEM 结果说明, 水热 合成法所得材料的粒径可控, 并可以轻松得到颗粒
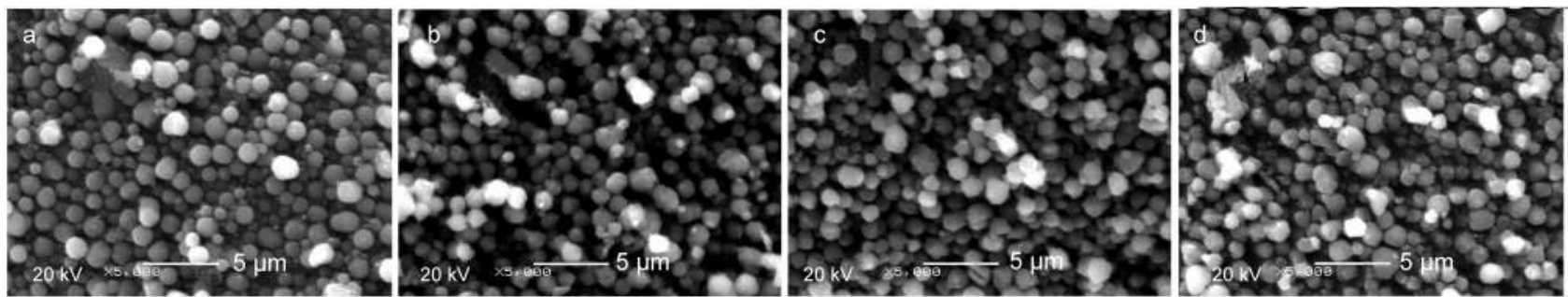

图 $4 \mathrm{TiO}_{2}$ 原料(a)在不同温度下热处理所得的 $\mathrm{Li}_{4} \mathrm{Ti}_{5} \mathrm{O}_{12}(\mathrm{~b}-\mathrm{d})$ 的 $\mathrm{SEM}$ 照片

Fig.4 SEM images of the spherical $\mathrm{TiO}_{2}(\mathrm{a})$ and $\mathrm{Li}_{4} \mathrm{Ti}_{5} \mathrm{O}_{12}$ calcined at different temperatures (b-d)

$T /{ }^{\circ} \mathrm{C}$ : (b) 800, (c) 850, (d) 900 
分布均匀一致的微米级材料, 这相比于传统固相法 所得材料粒径大且不均匀有一定的优势.

\section{$3.4 \mathrm{Li}_{4} \mathrm{Ti}_{5} \mathrm{O}_{12}$ 材料的粒径分析}

图 5 是水热反应的中间体经不同热处理温度 所得 $\mathrm{Li}_{4} \mathrm{Ti}_{5} \mathrm{O}_{12}$ 颗粒的激光粒度分布图. 从图中可以 看出, 当热处理温度从 $800{ }^{\circ} \mathrm{C}$ 增加到 $900{ }^{\circ} \mathrm{C}$, 所得 材料的粒径从 $0.99 \mu \mathrm{m}$ 增加到了 $1.99 \mu \mathrm{m}$. 经 $800{ }^{\circ} \mathrm{C}$ 处理的所得的 $\mathrm{Li}_{4} \mathrm{Ti}_{5} \mathrm{O}_{12}$ 粒径呈单一分布, 主要集中 在 $1 \mu \mathrm{m}$. 而 $850^{\circ} \mathrm{C}$ 和 $900{ }^{\circ} \mathrm{C}$ 所得的样品除了颗粒粒 径有所增大外, 粒径分布图上出现了双峰且峰宽逐 渐增大, 表明随着热处理温度的提高, 颗粒粒径逐 渐增大并出现一定程度的烧结且随温度的升高而 加重, 这与 SEM 图所示结果一致.

\section{$3.5 \mathrm{Li}_{4} \mathrm{Ti}_{5} \mathrm{O}_{12}$ 材料的电化学性能}

图 6 所示为各样品在室温下以 $35 \mathrm{~mA} \cdot \mathrm{g}^{-1}$ 的恒 电流进行充放电的放电容量-循环图. 从图中可以 看出, 首次循环中出现了不可逆容量, 但在接下来 的循环中并没有出现容量衰减, 热处理温度是影响

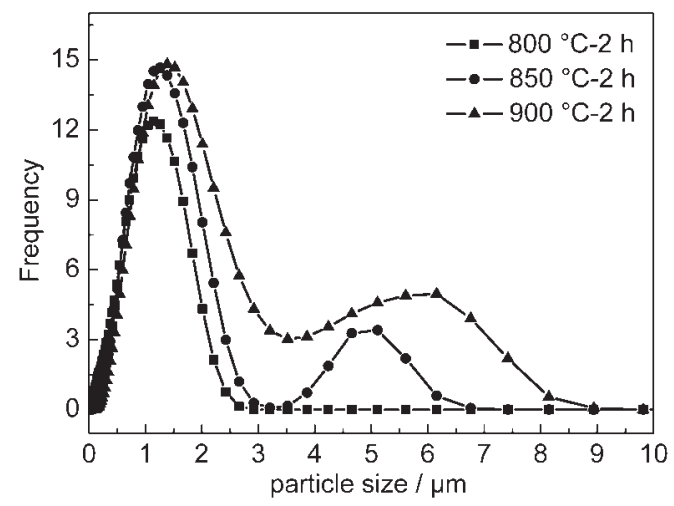

图 $5 \mathrm{Li}_{4} \mathrm{Ti}_{5} \mathrm{O}_{12}$ 材料粒度分布图

Fig.5 Particle size distribution of as-obtained $\mathrm{Li}_{4} \mathrm{Ti}_{5} \mathrm{O}_{12}$ materials

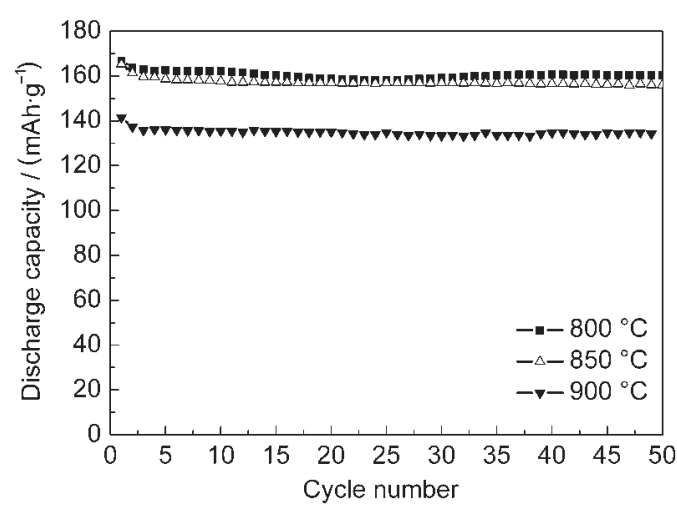

图 6 在 800-900 ${ }^{\circ} \mathrm{C}$ 下热处理所得的 $\mathrm{Li}_{4} \mathrm{Ti}_{5} \mathrm{O}_{12}$ 样品的 放电容量一循环图

Fig.6 Cycle performance of as-obtained $\mathrm{Li}_{4} \mathrm{Ti}_{5} \mathrm{O}_{12}$ after calcination at $800-900{ }^{\circ} \mathrm{C}$

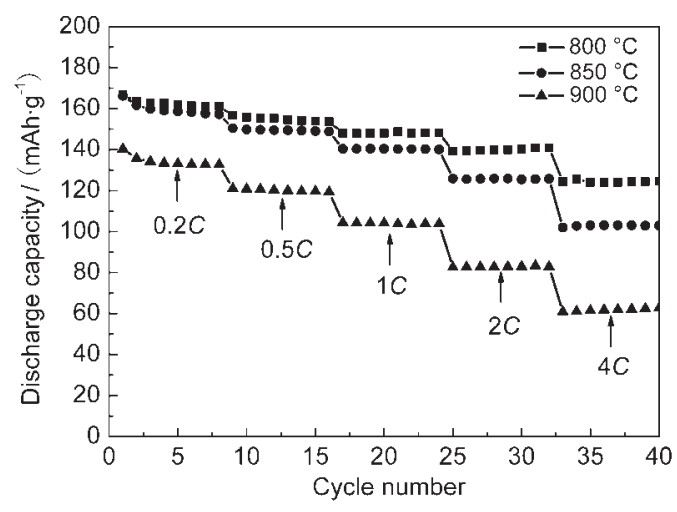

图 7 800-900 ${ }^{\circ} \mathrm{C}$ 热处理所得 $\mathrm{Li}_{4} \mathrm{Ti}_{5} \mathrm{O}_{12}$ 在不同放电电流密度 下的放电比容量-循环图

Fig.7 Cycling performance of the $\mathrm{Li}_{4} \mathrm{Ti}_{5} \mathrm{O}_{12}$ samples obtained between $800{ }^{\circ} \mathrm{C}$ and $900{ }^{\circ} \mathrm{C}$ at various current densities

所得材料放电容量的重要因素之一. 经 $800{ }^{\circ} \mathrm{C}$ 和 $850{ }^{\circ} \mathrm{C}$ 处理所得的两种 $\mathrm{Li}_{4} \mathrm{Ti}_{5} \mathrm{O}_{12}$ 材料放电容量相 近, 约为 $160 \mathrm{mAh} \cdot \mathrm{g}^{-1}$, 而经 $900^{\circ} \mathrm{C}$ 热处理所得的材 料放电容量要比 800 和 $850^{\circ} \mathrm{C}$ 所得样品的低, 只有 $135 \mathrm{mAh} \cdot \mathrm{g}^{-1}$ 左右, 这与不同热处理温度下所得的材 料粒径大小不同有关. $900{ }^{\circ} \mathrm{C}$ 热处理后颗粒烧结严 重, 粒径比 800 和 $850^{\circ} \mathrm{C}$ 的大, 对 $\mathrm{Li}^{+}$的扩散有所阻 碍, 使其不能在短时间内迅速扩散到颗粒内部, 使 材料的容量不能完全发挥出来. ${ }^{15,23-25}$ 但不同温度下 所得的三个样品都表现出较好的循环性能, 几乎无 衰减, 即使 50 个循环后三个材料的容量仍分别有 162、159和 $135 \mathrm{mAh} \cdot \mathrm{g}^{-1}$.

为了测试材料的倍率性能, 我们以 $35 \mathrm{~mA} \cdot \mathrm{g}^{-1}$ $(0.2 C)-720 \mathrm{~mA} \cdot \mathrm{g}^{-1}(4 C)$ 的电流密度对电池进行测 试, 结果见图 7. 从图中可以看出, $800^{\circ} \mathrm{C}$ 所制得的 $\mathrm{Li}_{4} \mathrm{Ti}_{5} \mathrm{O}_{12}$ 表现出了良好的倍率性能, 在 $720 \mathrm{~mA} \cdot \mathrm{g}^{-1}$ 的放电电流密度下仍可放出 $124 \mathrm{mAh} \cdot \mathrm{g}^{-1}$ 的容量. 这与之前 SEM 图和 PSD 图中所示样品由粒径均匀、 $1 \mu \mathrm{m}$ 左右的球形颗粒组成, 锂离子在其中需要的扩 散距离较短相一致. 而 850 和 $900{ }^{\circ} \mathrm{C}$ 所得样品由于 颗粒粒径随热处理温度升高逐渐增大, 同时烧结加 重, 大电流下充放电能力较差. 尤其是 $900{ }^{\circ} \mathrm{C}$ 下所 得的 $\mathrm{Li}_{4} \mathrm{Ti}_{5} \mathrm{O}_{12}$ 在 $720 \mathrm{~mA} \cdot \mathrm{g}^{-1}$ 的放电电流密度下只有 $60 \mathrm{mAh} \cdot \mathrm{g}^{-1}$ 左右的容量.

\section{4 结 论}

以自制球形 $\mathrm{TiO}_{2}$ 和 $\mathrm{LiOH}$ 溶液为反应物, 通过水 热反应制得 Li-Ti-O 中间体, 将所得中间体在 800$900{ }^{\circ} \mathrm{C}$ 热处理 $2 \mathrm{~h}$ 得到了纯相的微米级球形尖晶石 
$\mathrm{Li}_{4} \mathrm{Ti}_{5} \mathrm{O}_{12}$ 材料. 其中, $800{ }^{\circ} \mathrm{C}$ 所得的样品形貌均匀、 一致, 其可逆容量达到 $162 \mathrm{mAh} \cdot \mathrm{g}^{-1}$, 循环性能稳 定; 而 850 和 $900^{\circ} \mathrm{C}$ 热处理所得的材料颗粒粒径有 所增大, 其可逆容量较 $800{ }^{\circ} \mathrm{C}$ 所得的样品低, 大倍 率下进行充放电时, 容量衰减较快, 在 $720 \mathrm{~mA} \cdot \mathrm{g}^{-1}$ 的放电电流密度过下分别只有 100 和 $60 \mathrm{mAh} \cdot \mathrm{g}^{-1}$ 左 右的容量, 这与高温处理后颗粒粒径增大, 锂离子 扩散速度慢有关. 所得结果表明, 水热合成法所得 的 $\mathrm{Li}_{4} \mathrm{Ti}_{5} \mathrm{O}_{12}$ 材料颗粒均匀, 电化学性能好, 多次循环 几乎无衰减, 容量保持率达 $99 \%$ 以上. 该法与固相 法和溶胶-凝胶相比, 不需长时间进行高温热处理和 有污染昂贵的有机试剂, 且有利于大规模生产, 简 单易行.

\section{References}

(1) Ohzuku, T.; Ueda, A.; Yamamoto, N. J. Electrochem. Soc. 1995, $142,1431$.

(2) Abraham, K. M.; Pasquariello, D. M.; Willstaedt, E. B. J. Electrochem. Soc. 1990, 137, 743.

(3) Courtney, I. A.; Dahn, J. R. J. Electrochem. Soc. 1997, 144, 2045.

(4) Poizot, P.; Laruelle, S.; Grugeon, S.; Dupont, L.; Tarascon, J. M. J. Power Sources 2001, 97-98, 235.

(5) Poizot, P.; Laruelle, S.; Grugeon, S.; Dupont, L.; Tarascon, J. M. Nature 2000, 407, 496.

(6) Wang, G. J.; Gao, J.; Fu, L. J.; Zhao, N. H.; Wu, Y. P.; Takamura, T. J. Power Sources 2007, 174, 1109.

(7) Yao, J. W.; Wu, F. J. Funct. Mater. 2007, 38 (8), 1294. [姚经文, 吴 锋. 功能材料, 2007, 38 (8), 1294.]

(8) Pasquier, A. D.; Huang, C. C.; Spitler, T. J. Power Sources 2009, 186, 508

(9) Venkateswarlu, M.; Chen, C. H.; Do, J. S.; Lin, C. W.; Chou, T.
C.; Hwang, B. J. J. Power Sources 2005, 146, 204.

(10) Wang, D.; Ding, N.; Song, X. H.; Chen, C. H. J. Mater. Sci. 2009, 44, 198.

(11) Yang, J. W.; Zhong, H.; Zhong, H. Y.; Li, J.; Dai, Y. Y. J. Cent South Univ. 2005, 36 (1), 55. [杨建文, 钟 晖, 钟海云, 李 荐, 戴艳阳. 中南大学学报, 2005, 36 (1), 55.]

(12) Zaghib, K.; Simoneau, M.; Armand, M.; Gauthier, M. J. Power Sources 1999, 81-82, 300 .

(13) Guerfia, A.; Sevignya, S.; Lagacea, M.; Hovington, P.; Kinoshita, K.; Zaghib, K. J. Power Sources 2003, 119-121, 88.

(14) Bach, S.; Pereira-Ramos, J. P.; Baffier, N. J. Power Sources 1999, 81-82, 273.

(15) Jiang, C. H.; Ichihara, M.; Honma, I.; Zhou, H. S. Electrochim. Acta 2007, 52, 6470 .

(16) Li, J. R.; Tang, Z. L.; Zhang, Z. T. Electrochem. Commun. 2005, 7,894 .

(17) Li, Y.; Zhao, H. L.; Tian, Z. H.; Qiu, W. H.; Li, X. J. Alloy. Compd. 2008, 455, 471.

(18) Yu, Y.; Shui, J. L.; Chen, C. H. Solid State Commun. 2005, 135, 485.

(19) Nakahara, K.; Nakajima, R.; Matsushima, T.; Majima, H. J. Power Sources 2003, 117, 131.

(20) Hong, K. P.; Young, T. M.; Do, K. K.; Chong, H. K. J. Am. Ceram. Soc. 1996, 79, 2727.

(21) Allen, G. C.; Paul, M. Appl. Spectrosc. 1995, 49, 451.

(22) Gao, L.; Chen, J. Y.; Huang, J. H.; Yan, D. S. J. Inorg. Mater. 1995, 10 (4), 421. [高 濂, 陈锦元, 黄军华, 严东生. 无机材 料学报, 1995, $10(4), 421$.

(23) Lai, C.; Dou, Y. Y. ; Li, X.; Gao, X. P. J. Power Sources 2010, 195,3676

(24) Rahman, M. M.; Wang, J. Z.; Hassan, M. F.; Chou, S. L.; Wexler, D.; Liu, H. K. J. Power Sources 2010, 195, 4297.

(25) Yao, X. L.; Xie, S.; Chen, C. H.; Wang, Q. S.; Sun, J. H.; Li, Y. L.; Lu, S. X. Electrochim. Acta 2005, 50, 4076. 